\title{
Changes in the Serum Urate Level Can Predict the Development of Parkinsonism in the 6-Hydroxydopamine Animal Model
}

\author{
Mohammad Reza Sarukhani ${ }^{1} \cdot$ Hashem Haghdoost-Yazdi ${ }^{1}$ [D $\cdot$ Gilda Khandan-Chelarci ${ }^{2}$
}

Received: 17 December 2017 / Revised: 27 March 2018 / Accepted: 30 March 2018

(c) Springer Science+Business Media, LLC, part of Springer Nature 2018

\begin{abstract}
Epidemiological studies indicate that a higher plasma level of uric acid (UA) associates with the reduced risk of Parkinson's disease (PD). To confirm the role of UA as a biomarker for PD, we evaluated changes in the serum UA level in the 6-hydroxydopamine (6-OHDA)-induced hemiparkinsonism in rat. For this purpose, 6-OHDA was administered in the medial forebrain bundle by stereotaxic surgery. According to the apomorphine-induced rotational test, the increased intensity of behavioral symptoms as a function of time was associated with the further reduction of UA level. On the other hand, the level of UA increased in the midbrain of the injured hemisphere. The level of reduction in the serum UA level of rats with severe and moderate symptoms was significantly higher than that of rats with mild symptoms. The immunohistofluorescence and biochemical analyses showed that the serum UA level was also correlated with the death of tyrosine hydroxylase (TH)-positive neurons in the substantia nigra pars compacta $(\mathrm{SNc})$, reduced level of striatal dopamine, and severity of oxidative stress in the midbrain. The rats with mild symptoms also showed a significant decrease in TH-positive neurons and striatal dopamine level. These findings suggest a positive correlation between the level of reduction in the serum urate level and severity of 6-OHDA-induced Parkinsonism. In addition, our findings indicated that UA had no marked neuroprotective effects, at least at concentrations obtained in this study. On the other hand, UA was introduced as a biomarker for PD, as a significant decline was observed in the serum UA level of rats with mild behavioral symptoms but with significant dopaminergic cell death in the SNc.
\end{abstract}

Keywords Uric acid - 6-OHDA-induced hemiparkinsonism $\cdot$ Medial forebrain bundle $\cdot$ Apomorphine-induced rotational test $\cdot$ TH-positive neurons $\cdot$ Striatal dopamine level

\section{Introduction}

Parkinson's disease (PD) is a chronic and progressive neurodegenerative disorder, affecting about 6 million people worldwide [1]. The prevalence of PD is expected to double within the next 20 years, considering the increasing age of the population [2]. The most important characteristic of this disease is the progressive degeneration of dopaminergic (DA) neurons within the substantia nigra pars compacta $(\mathrm{SNc})$ of the midbrain. The pathophysiological mechanisms of DA degeneration remain unknown, although

Hashem Haghdoost-Yazdi

hhaghdoost@yahoo.com; hhaghdoost@gmail.com

1 Cellular and Molecular Research Center, Qazvin University of Medical Sciences, Qazvin 3414951414, Iran

2 Student Research Committee, Qazvin University of Medical Sciences, Qazvin, Iran mitochondrial dysfunction and oxidative stress are believed to play important roles in the events leading to DA neuronal death. Levodopa treatment removes dopamine deficiency and effectively improves movement-related symptoms. However, it cannot halt or retard progressive neurodegeneration, and side effects, such as dyskinesia, are developed in the long term [3-6].

PD is currently examined via clinical evaluation of extrapyramidal signs, such as tremor, rigidity, slowness or absence of voluntary movements, postural instability, and freezing. These symptoms occur when more than $70 \%$ of DA neurons are lost $[7,8]$. Therefore, early diagnosis of PD may help select and initiate the proper treatment to decelerate the neurodegenerative process and disease progression.

Identification of biomarkers for PD can be valuable in its early detection. The biomarkers also allow for a better follow-up of patients and facilitate objective measurements in clinical trials [9]. Nonetheless, research on the biomarkers 